\title{
Friendly regulates membrane depolarization induced mitophagy in Arabidopsis
}

\section{Authors}

Juncai Ma', Zizhen Liang', Jierui Zhao², Pengfei Wang', Wenlong Ma', Juan A. Fernandez Andrade ${ }^{3}$, Yonglun Zeng', Nenad Grujic², Liwen Jiang', Yasin Dagdas², Byung-Ho Kang'*

\section{Affiliations}

'School of Life Sciences, Centre for Cell \& Developmental Biology and State Key Laboratory of Agrobiotechnology, The Chinese University of Hong Kong, Shatin, New Territories, Hong Kong, China. ${ }^{2}$ Gregor Mendel Institute (GMI), Austrian Academy of Sciences, Vienna BioCenter (VBC), Vienna, Austria. ${ }^{3}$ Department of Physiology and Pharmacology, Western University, London, Ontario, Canada.

*e-mail: bkang@cuhk.edu.hk, yasin.dagdas@gmi.oeaw.ac.at

\section{Abstract}

The oxidative environment within the mitochondria makes them particularly vulnerable to proteotoxic stress. To maintain a healthy mitochondrial network, eukaryotes have evolved multitiered quality control pathways. If the stress cannot be alleviated, defective mitochondria are selectively removed by autophagy via a process termed mitophagy. Despite significant advances in metazoans and yeast, in plants, the molecular underpinnings of mitophagy are largely unknown. Here, using time-lapse imaging, electron tomography and biochemical assays, we show that uncoupler treatments cause loss of mitochondrial membrane potential and induce autophagy in Arabidopsis. The damaged mitochondria are selectively engulfed by autophagosomes that are ATG5 dependent and labelled by ATG8 proteins. Friendly, a member of the Clustered Mitochondria protein family, is recruited to the damaged mitochondria to mediate mitophagy. In addition to stress, mitophagy is also induced during de-etiolation, a major cellular transformation during photomorphogenesis that involves chloroplast biogenesis. De-etiolation triggered mitophagy regulates cotyledon greening, pointing towards an inter-organellar cross-talk mechanism. Altogether our results demonstrate how plants employ mitophagy to recycle damaged mitochondria during stress and development. 


\section{Introduction}

2 Mitochondria are highly dynamic double-membraned organelles that function as cellular

3 powerhouses. They generate energy via oxidative phosphorylation (OXPHOS) and mediate

4 the synthesis of essential macromolecules such as iron-sulfur clusters ${ }^{1,2}$. One of the by-

5 products of the oxidative environment in mitochondria is generation of toxic reactive oxygen

6 species that damage mitochondrial DNA, lipids and proteins. In addition, although most of the

7 mitochondrial proteins are encoded by nuclear genes, I3 subunits of the oxidative

8 phosphorylation complexes are still encoded by the mitochondrial genome. As the inter-

9 genome coordination could be disrupted, and one cell could have thousands of times more

10 copies of mitochondrial genome than the nuclear genome; imbalances in stoichiometries of

11 these multi-subunit OXPHOS complexes trigger proteotoxic stress ${ }^{3,4}$. To overcome these

12 challenges and maintain a healthy mitochondrial network, eukaryotes have evolved multi-

13 tiered and interconnected mitochondrial quality control pathways ${ }^{3}$.

One of the major mitochondrial quality control pathways is mitophagy, the selective removal of damaged or superfluous mitochondria via autophagy. As many players involved in mitophagy have been associated with disease, and mitophagy allows us to visualize selective engulfment of an organelle into an autophagosome, mitophagy is one of the best studied signalling mechanisms in metazoans ${ }^{5-8}$. One of the hallmarks of damaged mitochondria is loss of membrane potential ${ }^{3}$. Various chemical protonophores such as carbonyl cyanide ptrifluoro-methoxyphenyl hydrazone (FCCP) or 2,4-dinitrophenol (DNP) have been used to induce mitochondrial membrane depolarization and mitophagy 9 . Loss of mitochondrial membrane potential leads to the stabilization of PINKI on mitochondrial outer membrane. PINKI phosphorylates ubiquitin and activates Parkin on mitochondrial membrane for polyubiquitination of various outer membrane proteins. This creates a positive feedback loop that results in recruitment of various selective autophagy receptors such as $p 62$, Optineurin or NDP52 to recruit the damaged mitochondria into autophagosomes for their subsequent degradation ${ }^{7,10,11}$. Although much has been learnt about mitophagy in metazoans, molecular players that mediate mitophagy in plants is currently unknown ${ }^{2,12}$.

Mounting evidence suggests plant mitochondria are also recycled by selective autophagy ${ }^{2,13}$. However, likely influenced by harbouring another endosymbiotic organelle, 33 plants lack homologs of known mitophagy receptors and regulators ${ }^{12}$. Also, so far, most 
34 studies used genetic and biochemical assays to analyse mitochondrial turnover in plants. Cell 35 biological tools that would allow us to visualize different stages of mitophagy have not been established. Here, we studied uncoupler induced mitophagy in the model plant Arabidopsis thaliana. We used live cell imaging and electron tomography to visualize the engulfment of the damaged mitochondria by mitophagosomes. We supported our cell biological findings with autophagic flux assays to show autophagy regulates recycling of damaged mitochondria. We also showed that Friendly (FMT) protein that has been linked to the regulation of mitochondria dynamics is recruited to mitochondria upon damage. Consistently, fmt mutants have defects in formation of mitophagosomes and mitochondrial turnover. Finally, we demonstrate that de-etiolation also leads to accumulation of compromised mitochondria and induces mitophagy. Altogether, our findings establish a cell biological and biochemical platform to further dissect mitophagy and reveal a molecular player that is essential for mitophagy in plants.

\section{Results}

Uncoupler treatments induce accumulation of depolarized mitochondria in Arabidopsis root cells.

Uncouplers such as DNP and FCCP perturb the electrochemical potential of inner mitochondrial membrane, triggering mitochondrial recycling in mammalian cells ${ }^{14}$. To test whether these compounds also affect mitochondria in plant root tip cells, we incubated Arabidopsis seedlings expressing mitochondrion-targeted GFP (Mito-GFP) in liquid MS medium containing DNP or FCCP (Fig. I). For consistency, our live cell imaging and electron microscopy/tomography of mitochondria were limited to cortex cells in the root elongation zone. However, loss of membrane potential and mitochondrial recycling were observed in all cell types. To differentiate depolarized mitochondria, we pre-stained root cells with tetramethylrhodamine ethyl ester (TMRE), a fluorescent dye sensitive to membrane potential ${ }^{15}$. Normal mitochondria are seen as yellow puncta from dual fluorescence emitted from GFP and

61 TMRE, while depolarized mitochondria will be green as TMRE will not fluoresce upon membrane depolarization.

Under normal conditions, depolarized mitochondria were rare in Mito-GFP roots (Fig. la). When Mito-GFP roots were incubated with DNP $(50 \mu \mathrm{M})$ for I hr, numbers of depolarized mitochondria increased significantly (Fig. Ia,c). Inactivation of a core macroautophagy gene, ATG5, in the Mito-GFP line (atg5-I::Mito-GFP) led to accumulation of 
more depolarized mitochondria in DNP-treated as well as untreated roots, indicating that removal of depolarized mitochondria requires ATG5 (Fig. Ib,d). Addition of Concanamycin A (ConA), an inhibitor of vacuolar $\mathrm{H}^{+}$-ATPase that disrupts protein transport to the vacuole ${ }^{16}$, led to further build-up of mitochondria lacking membrane potential in Mito-GFP roots, indicating vacuole is the final destination for these depolarized mitochondria. Importantly, ConA did not lead to a similar build-up of depolarized mitochondria in atg5- I::Mito-GFP roots (Fig. Id, Extended Data Fig. I). These observations agree with the inhibition of autophagy by ConA ${ }^{17,18}$ and suggest that depolarized mitochondria are recycled via the macroautophagy machinery in Arabidopsis root cells.

FCCP was a more potent uncoupler than DNP, depolarizing almost all mitochondria at a lower concentration after I hr (Fig. I). In the following analyses, however, we employed DNP to trigger mitophagy, because its slower action facilitated the monitoring of the mitophagy dynamics via cell biological and biochemical assays.

\section{Uncoupler treatments induce autophagy}

To examine whether autophagosome formation is induced following the uncoupler stress, we visualized a member of the Arabidopsis ATG8 family, ATG8e, fused with a YFP (YFPATG8e). ATG8 is widely used as a marker for autophagosomes in all eukaryotes ${ }^{19,20}$. Under normal conditions we rarely observed puncta; most of the ATG8 signal was diffuse. However, The YFP-ATG8e foci multiplied in root cells after I hr of DNP treatment, and their numbers continued to increase at later time points (Fig. 2a,b). Upon activation of autophagy, ATG8 becomes conjugated to phosphatidylethanolamine (PE) by a complex containing ATG5, and affixed to the limiting membrane of autophagosomes ${ }^{17}$. The lipidated form of ATG8 runs faster in western blots, and the ratio between lipidated and unlipidated ATG8 is used as a proxy to measure autophagy ${ }^{16}$. Immunoblot analyses with ATG8 antibody revealed only a faint upper band in untreated wild type (WT) cells (Fig. 2c). This band became more abundant by DNP treatment, especially in the membrane fraction. Samples incubated with phospholipase D (PLD) or samples from atg5-I mutants lacked the upper band (Fig. 2d). Altogether, these results suggest that ATG8 lipidation is induced upon DNP treatment in an ATG5 dependent manner.

To further confirm that the uncoupler stress induces autophagosome formation and vacuolar delivery, we performed GFP cleavage assay with the YFP-ATG8e line and atg5-I mutant line expressing an mCherry-ATG8e chimeric protein (mCherry-ATG8e::atg5-I). A 
100 free YFP polypeptide was detected in YFP-ATG8e samples and its amount increased over 101 time with a concomitant drop in YFP-ATG8e (Fig. 2e,f). No free mCherry was discerned in 102 the immunoblot of mCherry-ATG8e::atg5-I by an anti-mCherry antibody (Fig. 2e). Excitingly, 103 DNP treatment did not affect the autophagic flux of an aggrephagy receptor, Neighbor of 104 BRCAI (NBRI) $)^{2 I}$ (Fig. 2e). These data suggest that uncoupler treatment activates a selective 105 autophagy pathway to recycle depolarized mitochondria.

Damaged mitochondria are selectively engulfed by autophagosomes in uncoupler treated root cells.

We then examined if DNP induced autophagosomes were indeed engulfing mitochondria by staining YFP-ATG8e root cells with MitoTracker Red (MTR). Under a confocal microscope,

111 YFP-positive puncta were seen in the vicinity of mitochondria (Fig. 3a). In higher magnification 112 micrographs, we were able to identify ATG8e-specific fluorescent structures resembling open 113 pouches that contain mitochondria (arrowheads, Fig. 3b). We were also able to observe 114 mitochondria that were entirely surrounded by YFP-ATG8e rings (white arrow, Fig. 3b). In 115 time-lapse live cell imaging, YFP-ATG8e pockets partially enclosing a mitochondrion were seen to grow, eventually encapsulating the mitochondrion over 300 seconds (Fig. 3c). These

117 autophagic compartments (i.e., mitophagosomes) were approximately I-2 $\mu \mathrm{m}$ in diameters 118 and each carried a single mitochondrion. MTR stains depolarized mitochondria better than 119 TMRE but does not concentrate in mitochondria with no membrane potential ${ }^{22}$. In this vein, 120 empty autophagosomes matching the size of mitophagosomes (grey arrow in Fig. 3b) in our 121 micrographs could correspond to mitophagosomes carrying mitochondrial corpses.

122 A time-lapse movie revealed that a small YFP-ATG8e puncta arose near MTR stained mitochondria and elongated to be a semicircle capturing a mitochondrion. This process took 124 about 10 mins (Extended Data Fig. 2a). Another video documented an incomplete 125 mitophagosome that expanded to enclose a mitochondrion fully (Extended Data Fig. 2b). 126 Elongating tips of phagophores stayed in contact with the mitochondrial surface throughout 127 their growth (Extended Data Fig. 3a). From our live cell microscopy data, we estimated that 128 it takes about 15 min for mature mitophagosomes to develop from an initial YFP-ATG8 spot 129 on a mitochondrion.

130 We then cryofixed root samples and performed transmission electron microscopy (TEM) 131 analysis. Mitochondria in control samples had smooth cristae that are evenly dispersed in the 132 matrix (Fig. 3d,g). By contrast, mitochondria in DNP treated cells had electron-dense 
133 precipitates in their matrix, some of which were engulfed by double membraned 134 mitophagosomes (Fig. 3 e-k). Serial section TEM of mitophagosomes showed that one 135 mitochondrion was contained per autophagosome, and no other organelles were identified in 136 autophagosomes (Extended Data Fig. 3b,c), in agreement with the live cell imaging results (Fig. 137 3b,c). Electron tomography analysis revealed that mitochondria sequestered in 138 mitophagosomes have more dark precipitate but less cristae in the matrix than free 139 mitochondria (Fig. 3g-k). Arabidopsis atg5-I root cells had many mitochondria exhibiting the 140 signs of the internal precipitates, but they were not associated with mitophagosomes (Fig. 3I). 141 Altogether these results show that plant cells selectively recycle damaged mitochondria via 142 autophagy that involves ATG5.

143 To further investigate how mitochondria are degraded during uncoupler induced 144 mitophagy, we used immunoblot assays to assess the levels of various mitochondrial proteins 145 in Arabidopsis WT and atg5-I mutant lines. We treated Arabidopsis seedlings with DNP for one 146 to four hours (DI-D4). To distinguish between uncoupler induced mitophagy and bulk 147 autophagy, we used nitrogen starvation as control (Extended Data Fig. 4). In contrast to the 148 endoplasmic reticulum protein Cycloartenol-C24-methyl transferase (SMTI), levels of outer 149 mitochondrial membrane (OMM) proteins peripheral-type benzodiazepine receptor (PBR) 150 and voltage dependent anion channel I (VDACI); inner mitochondria membrane (IMM) 151 proteins cytochrome oxidase subunit II (COXII) and L-galactono-I,4-lactone dehydrogenase $152(\mathrm{GLDH})$, and mitochondria matrix (MM) protein isocitrate dehydrogenase (IDH) were all 153 reduced upon uncoupler treatment (Fig. 4a). The reduction in protein levels were due to 154 vacuolar degradation as addition of ConA prevented the degradation (Fig. 4b). Furthermore, 155 the degradation was dependent on ATG5, since protein levels did not change significantly in 156 DNP treated atg5-I mutant specimens (Fig. 4a,c). Interestingly, OMM proteins showed higher 157 levels of degradation in contrast to IMM and matrix proteins. Since, previous studies in 158 mammalian cells showed OMMs could also be degraded via the proteasome ${ }^{23}$, we checked 159 OMM protein levels of DNP treated cells following proteasome inhibition by MGI32. Adding 160 MGI32 indeed stabilized only OMM proteins but not IMM or matrix proteins (Fig. 4d). 161 Considered together with normalized quantification of protein levels (Fig. 4e,f), these 162 experiments suggest upon loss of membrane potential, proteasome and autophagy cooperate 163 to degrade OMM proteins, whereas IMM and matrix proteins are primarily degraded by 164 autophagy. 


\section{Friendly is essential for uncoupler induced mitophagy.}

We then wanted to identify molecular players that mediate mitophagy in plants. Previous studies have shown that for both ubiquitin dependent and independent mitophagy pathways, mitochondrial network needs to be fragmented ${ }^{24,25}$. In Arabidopsis, Friendly (FMT), a clustered mitochondria (CLU) family protein, has been shown to play important roles in mitochondrial

171 dynamics $^{26}$. However, whether it plays a role in mitophagy wasn't addressed. YFP-FMT

172 exhibited a diffuse cytosolic pattern under normal conditions. Interestingly, upon DNP treatment, YFP-FMT localized to puncta that colocalized with mitochondria (Fig. 5a). Pull

174 down experiments showed that uncoupler treatment led to specific association of YFP-FMT 175 with ATG8, suggesting that YFP-FMT localizes to mitophagosomes (Fig. 5b). This prompted 176 us to analyse mitophagy in fmt mutant. Consistent with our previous findings presented in Fig.

177 3, live cell imaging of GFP-tagged mitochondria in mCherry-ATG8e expressing WT cells 178 showed ATG8e labelled vesicles engulfed mitochondria upon uncoupler treatment. However, 179 in fmt mutant, although ATG8 puncta were associated with the mitochondria, we did not 180 observe engulfment of mitochondria within autophagosomes (Fig. 5c). Further analyses of 181 mitophagosomes in the fmt mutant using TEM revealed aberrant mitophagosomes with disconnected edges (Fig. 5c).

183 To further test the role of FMT in mitophagy, we performed live cell imaging and immunoblot based autophagic flux experiments. Staining of depolarized mitochondria with TMRE upon uncoupler treatments revealed accumulation of damaged mitochondria in the cytosol of fmt mutants (Fig. 6a). Quantification of depolarized mitochondria showed fmt mutants accumulated significantly more mitochondria in contrast to WT cells (Fig. 6b). Furthermore, morphometric analyses of mitochondria in TEM micrographs from WT, fmt and atg5-I mutants showed that mitochondria were significantly larger in fmt and atg5-I mutants (Extended Data Fig. 5). Finally, immunoblot analyses using mitochondrial compartment specific antibodies also showed a delay of mitochondrial protein degradation in fmt mutant (Fig. 6c). Comparative analyses of the polypeptide intensities indicated that although fmt mutant had a significant defect in mitochondrial protein recycling (Fig. 6d), it was not as severe as the atg5-

194 I mutant (Fig. 4d,e), suggesting, in the absence of FMT, compensatory pathways prevent 195 accumulation of the toxic damaged mitochondria in the cell. Altogether these experiments 196 suggest FMT is required for mitophagy in plants. 


\section{Cotyledon greening during de-etiolation is affected in atg5-I mutant seedlings.}

In germinating seeds, under darkness, proplastids transform into etioplasts that are characterized by paracrystalline arrays of prolamellar bodies in their stroma ${ }^{27}$. The etioplast quickly transforms into the chloroplast upon exposure to light, a major developmental transition termed de-etiolation. Since mitochondrial and chloroplast functions are tightly interconnected, we hypothesized that mitochondrial population may also undergo remodelling during deetiolation. First, we monitored greening of cotyledons when dark grown seedlings were exposed to light. Green pigment levels increased gradually over a $12 \mathrm{hr}$ period, with a significant rise at eight hours after illumination (Fig. 7a,b). We then measured amounts of mitochondrial proteins in greening cotyledon cells and showed that their levels also drop at $8 \mathrm{hr}$ time point, suggesting an accelerated removal of mitochondria (Fig 7c). Consistently, mitophagosomes were most frequently detected in cotyledon cell sections from 6 and $8 \mathrm{hr}$ samples under TEM (Fig. 7d). Cotyledon greening was severely affected in atg5-I mutant seedlings and no mitophagosomes were discerned in their cotyledon cells (Fig. 7 a,b,e). These results indicate de-etiolation is a physiological stress condition for mitochondria where mitophagy is induced to mediate mitochondrial turnover that underlies light activated cotyledon development.

\section{Discussion}

Research in the last decade has transformed autophagy from a bulk degradation system to a highly selective cellular quality control pathway that rapidly removes toxic or superfluous macromolecules ${ }^{28,29}$. Especially organelles that get damaged due to metabolic and physiological stress conditions are mainly recycled via distinct selective autophagy pathways ${ }^{6}$. Consistently, in all the eukaryotes tested so far, autophagy is essential for adapting to environmental changes $^{12,30}$. However, despite significant advances made in metazoan selective autophagy field, how plants recycle their organelles are still mostly unknown. Although the core autophagy machinery that mediates formation of the autophagosome is highly conserved in plants, selective autophagy receptors and adaptors that are responsible for recognition and recruitment of damaged organelles to the autophagosomes are not well conserved ${ }^{12}$. For example, there are up to eight different receptor proteins and dozens of accessory proteins that have been shown to mediate various mitophagy pathways in mammalian cells ${ }^{3}$. Homologs of most of those proteins are lacking in plant genomes, implying plant mitophagy have followed a different evolutionary path, likely due to the presence of another endosymbiont in the cell. 
Here, we have established a detailed toolbox to study mitophagy in plants. We have shown that protonophore uncouplers specifically induce mitophagy similar to metazoans. Our

231 findings also revealed high levels of mitophagy during de-etiolation, a fundamental

232 developmental step that allows plants to survive day light after germination. Excitingly, our 233 studies also revealed a molecular player, the Friendly protein, that is essential for mitophagy.

\section{Mitochondrial membrane potential is closely monitored by mitochondrial quality} control pathways

As the primary producer of ATP in eukaryotic cells, healthy mitochondria must maintain the electrochemical potential. It is conceivable that the loss/reduction in the potential serves as a mark for the mitophagy machinery to recognize malfunctioning mitochondria. For example, in the PINKI/Parkin-dependent mitophagy pathway of mammalian cells, a mitochondrion-localized protein kinase, Pinkl, is stabilized when the membrane potential drops and this leads to the activation of downstream effectors ${ }^{31}$. In $C$. elegans sperm mitochondria are rapidly removed from the oocyte via mitophagy upon fertilization ${ }^{32,33}$. 243 Although the underlying mechanism of depolarization is still unknown, the paternal mitochondria that is recycled by mitophagy loses its membrane potential prior to mitophagy ${ }^{15}$.

245 Our uncoupler treatments clearly demonstrated that membrane potential serves as a proxy 246 for mitochondrial health across different organisms and loss of membrane potential triggers mitophagy. However, how plants tag depolarized mitochondria for mitophagy needs to be

248 further investigated.

Mitochondria in mammalian and yeast cells undergo cycles of fusion and fission. This mitochondrial dynamics collaborate with mitophagy; fusion can rescue damaged mitochondria 252 by diluting their injuries while fission singles out aberrant mitochondria for mitophagy. It was 253 shown that mitochondria fusion is inhibited when the mitochondrial membrane potential is 254 dissipated by uncouplers ${ }^{34}$. Mitochondria in Arabidopsis root cells are mostly round, indicating 255 that mitochondria fission dominates over fusion ${ }^{35}$. Therefore, our system is not suited for investigating the link between mitochondria's membrane potential and their fusion/fission dynamics. Some plant cells including seed cells after germination or shoot apical meristem cells have elongated mitochondria constituting a network ${ }^{36,37}$. These cells are better for testing whether mitochondria fragment in response to uncoupler stresses and studying roles of 
262 Ultrastructural features of compromised mitochondria and mitophagosomes in

263 Arabidopsis root cells.

Mitochondria with dark aggregates and shrivelled cristae in the matrix appeared after incubation with uncouplers. They were also abundant in de-etiolating cotyledon cells and fmt mutant cells (Fig. 3 and Extended Data Fig. I). These impaired mitochondria were rare in DMSO control samples but frequently observed in atg5-I mutant samples in TEM images. Considering that they are specifically targeted by and enclosed in mitophagosomes, they correspond to depolarized mitochondria being recycled. Sperm mitochondria in cryofixed $C$. 271 elegans oocytes also exhibited similar ultrastructural features ${ }^{15,38}$. However, we did not observe large ruptures in mitochondrial membranes reported in TEM analysis of mammalian mitophagy where cells were preserved by chemical fixation ${ }^{39}$.

An intriguing feature that we noticed in our TEM analysis is that tips of the elongating phagophores were in contact with the mitochondrial surface (Fig. 3e, arrowheads), This observation is consistent with the YFP-ATG8e fluorescence that expanded tightly over mitochondria in the time-lapse recordings (Fig. 3c). The affinity between the autophagosome membrane and mitochondria explains why mitophagosomes usually have one damaged mitochondrion, and they do not capture other organelles. It also suggests specific proteinprotein interactions regulate the two membranes, which need to be investigated further.

\section{FMT is essential for mitophagy in plants.}

FMT is required for normal mitochondria distribution in the plant cell, possibly regulating association between individual mitochondria before fusion ${ }^{26}$. We have shown that DNPinduced mitochondria recycling is affected in fmt mutant cells, and FMT associates with ATG8 upon mitochondrial damage. These data suggest that FMT associates with autophagosomes during mitophagy. Because FMT is a protein shuttling between the cytosol and mitochondria, it is tempting to speculate that FMT may participate in an autophagy receptor or adaptor complex through which the core autophagy machinery is recruited to mitochondria. It is also

293 interaction with the healthy mitochondria, and excessive accumulation of FMT triggers the 294 onset of mitophagy. 
FMT is a highly conserved protein with orthologs in evolutionarily distant eukaryotes including yeast and metazoans ${ }^{40}$. fmt mutants exhibit similar phenotypes where mitochondria

298 form large clusters next to nucleus. The Drosophila FMT homolog Clueless positively regulates PINKI/Parkin dependent mitophagy by suppressing mitochondrial fusion ${ }^{41}$. Recent studies have shown that mammalian FMT homolog CLUH could bind RNA to form granules.

$301 \mathrm{CLUH}$ granules regulate translation of mRNAs linked to metabolic activity and regulate mitophagy and metabolic reprogramming ${ }^{42,43}$. Plants lack PINKI/Parkin homologs, so whether

303 FMT regulates mitophagy by forming stress activated granules or via a PINK/Parkin-like 304 pathway need to be investigated further. Identification of FMT interacting proteins and RNA during nutrient starvation or uncoupler treatments could help us understand the role of FMT in mitophagy and mitochondrial quality control.

Mitochondrial recycling mediate organellar reprogramming during de-etiolation.

Reprograming the mitochondrial functions in response to nutrient availability is critical 312 are coordinated for homeostasis of cellular energy levels and redox status ${ }^{45,46}$. Our results 313 from greening Arabidopsis cotyledons indicated that de-etiolation involves a wave of 314 mitochondrial turnover, probably for rewiring mitochondrial metabolic network for adapting 315 to light condition and salvaging raw materials for chloroplast biogenesis.

In skotomorphogenic seedlings, nutrients reserved in the seed are mobilized to sustain growth, and mitochondria are required for the anabolic processes. When light is available, the seedlings become photosynthetically active and capable of autotrophic growth. It was shown that the electron transport chain in mitochondria is slowed down in de-etiolating wheat leaves and chloroplasts and mitochondria are functionally more intertwined ${ }^{47}$. Accumulation of mitophagosomes and rapid changes in mitochondrial protein levels in the greening cotyledon suggest a cannibalization of pre-existing mitochondria. We speculate that the developmentally programmed mitophagy could facilitate modulation of the mitochondrial pool and biosynthesis of macromolecules for constructing new organelles. The lack of mitophagosomes and the delay in greening of the atg5-I mutant cotyledon agrees with the notion. Altogether, our findings on greening cotyledons present a clear example of inter-organelle communication, 
and how mitochondria-chloroplast crosstalk could underlie a major developmental transition in plants.

\section{Methods}

\section{Materials and plant growth conditions}

333 All the chemicals were purchased from Sigma-Aldrich (http://www.sigmaaldrich.com) or Thermo334 Fisher (https://www.thermofisher.com) unless specified. YFP-ATG8e, mCherry-ATG8e, atg5-I, Mito335 GFP, Mito-GFP::fmt and FMT-YFP seeds were described previously26,48,49. mCherry-ATG8e::atg5-I, 336 Mito-GFP $\times$ mCherry-ATG8e and Mito-GFP $\times$ mCherry-ATG8e::fmt was obtained by crossing the 337 previously established lines 26,50 . All transgenic lines were genotyped by PCR and homozygous lines 338 were isolated before experiments. All Arabidopsis seeds were surface-sterilized and geminated on 1/2

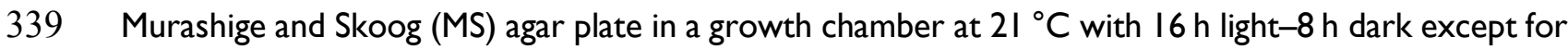
340 de-etiolation experiments where seedlings were geminated under darkness.

\section{Protein extraction and immunoblot analysis}

342 Isolation of mitochondrial membrane proteins and GFP cleavage assay were carried out as described 343 previously ${ }^{48,51}$. Briefly, seven-day-old Arabidopsis seedlings were incubated in DMSO, $50 \mu M$ DNP, 50

$344 \mu \mathrm{m} \mathrm{MGI} 32$ or I $\mu \mathrm{m}$ ConA with indicated times in liquid half MS medium when necessary. Seedlings 345 for the nitrogen starvation experiments were germinated on half MS medium agar plate and then 346 transfer to liquid half MS medium without nitrogen for I day. All the protein samples were subjected to I5\% SDS-PAGE. Primary and secondary antibodies were diluted in Ix phosphate buffered saline (PBS). Antibodies against GFP (Abcam), YFP (Agrisera), mCherry (Abcam), ATG8 (Agrisera), voltagedependent anion channel I (VDACI; Agrisera), peripheral-type benzodiazepine receptor (PBR; PhytoAB), cytochrome oxidase subunit II (COXII; Agrisera), L-galactono-I,4-lactone dehydrogenase (GLDH; Agrisera, PhytoAB) and isocitrate dehydrogenase (IDH; Agrisera), Cycloartenol-C24-methyl transferase (SMTI; Agrisera), Coatomer subunit gamma (Sec2lp; Agrisera) were obtained from the indicated sources. We performed Student's $t$ test (one-tailed and unpaired test) with the triple replicate immunoblot data and the quantification of band intensities was performed using ImageJ

356 Software). Representative of at least three independant immunoblot results were shown in the figures.

\section{ATG8 delipidation assay}

358 Protein extraction methods for ATG8-delipidation were described previously52. Seven-day-old 359 Arabidopsis seedlings were incubated in $50 \mu \mathrm{M}$ DNP for 2 hours before protein extraction. The total 360 plant lysates were extracted in lysis buffer $[50 \mathrm{mM}$ Tris- $\mathrm{HCl}(\mathrm{pH} 8.0), 150 \mathrm{mM} \mathrm{NaCl}, 0.5 \mathrm{mM}$ 
361 Ethylenediaminetetraacetic acid (EDTA) and Ix Complete Protease Inhibitor Cocktail] and then

362 centrifuged at $14000 \mathrm{rpm}$ for $10 \mathrm{~min}$ at $4{ }^{\circ} \mathrm{C}$. The supernatant was centrifuged at $100000 \mathrm{~g}$ for $\mathrm{I} \mathrm{h}$, 363 with the membrane pellet then solubilized in lysis buffer containing $0.5 \%(\mathrm{v} / \mathrm{v})$ Triton $X-100$. The 364 solubilized membrane samples were incubated at $37^{\circ} \mathrm{C}$ for I h with 250 unit $/ \mathrm{ml}$ of phospholipase D 365 (PLD) or an equal volume of its buffer. Protein samples were subjected to I5\% SDS-PAGE in the 366 presence of $6 \mathrm{M}$ urea and analyzed by immunoblot with anti-ATG8 antibody.

\section{Immunoprecipitation}

368 Protein extraction and immunoprecipitation were performed as described previously ${ }^{48}$. Seven-day-old 369 Arabidopsis seedlings were incubated in $50 \mu$ M DNP for 2 hours before protein extraction. Total plant 370 lysates were centrifuged at $14000 \mathrm{rpm}$ for $10 \mathrm{~min}$ at $4{ }^{\circ} \mathrm{C}$. The supernatant was prepared in lysis buffer 371 (I0 mM Tris/ $\mathrm{HCl}$ at pH 7.4, I $50 \mathrm{mM} \mathrm{NaCl}, 0.5 \mathrm{mM}$ EDTA, $5 \%$ glycerol, $0.2 \%$ Nonidet P-40, and $2 \mathrm{mM}$ 372 dithiobis [succinimidyl propionate] containing Ix Complete Protease Inhibitor Cocktail) and then 373 incubated with GFP-TRAP agarose beads (ChromoTek) for 2 hours at $4^{\circ} \mathrm{C}$. The beads were washed 374 five times $\left(4^{\circ} \mathrm{C}\right)$ in wash buffer $(10 \mathrm{mM}$ Tris/ $\mathrm{HCl}, \mathrm{pH} 7.5,150 \mathrm{mM} \mathrm{NaCl}$, and $0.5 \mathrm{mM}$ EDTA with Ix 375 Complete Protease Inhibitor Cocktail) and then eluted by boiling in 2x SDS sample buffer. Samples 376 were separated by SDS-PAGE and analyzed by immunoblot using indicated antibodies.

\section{Confocal microscopy and image processing}

379 Confocal fluorescence images were acquired using the Leica SP8 laser scanning confocal system with 380 a $63 x$ water lens. Seven-day-old Arabidopsis seedlings were incubated in DMSO, $50 \mu M$ DNP, $10 \mu M$ 381 FCCP or $0.5 \mu \mathrm{M}$ ConA with indicated times in liquid half MS medium when necessary before imaging. 382 Tetramethylrhodamine ethyl ester (TMRE) and MitoTracker Red (MTR) were used to stain Arabidopsis 383 root cell mitochondria at $500 \mathrm{~nm}$ for 10 mins. A sequential acquisition was applied when observing 384 fluorescent proteins. Images were processed with Photoshop CC (https://www.adobe.com) and 385 performed Student's $t$ test (one-tailed and unpaired test) with Microsoft Excel 2016 386 (https://www.microsoft.com/). The graphs were prepared with Prism8 (https://www.graphpad.com).

\section{TEM analysis, electron tomography, and $\mathbf{3 d}$ modeling}

388 For TEM samples preparation, high-pressure freezing, freeze substitution, resin embedding, and 389 ultramicrotomy were performed as described previously53,54. In brief, Seven-day-old Arabidopsis 390 seedlings were incubated in DMSO or $50 \mu \mathrm{M}$ DNP for indicated times and then rapidly frozen with 391 an HPMI00 high-pressure freezer (Leica Microsystems). The samples were freeze-substituted at $-80^{\circ} \mathrm{C}$ 392 for $72 \mathrm{~h}$, and excess OsO4 was removed by rinsing with precooled acetone. After being slowly 393 warmed up to room temperature over $48 \mathrm{~h}$, root samples were separated from planchettes and 394 embedded in Embed-8I2 resin (Electron Microscopy Sciences). Thin sections ( $100 \mathrm{~nm}$ thick) prepared 
395 from sample blocks of each time point were examined with a Hitachi 7400 TEM (Hitachi-High

396 Technologies) operated at $80 \mathrm{kV}$.

397 For dual-axis tomography analysis, semi-thick sections $(250 \mathrm{~nm})$ were collected on formvar-coated

398 copper slot grids (Electron Microscopy Sciences) and stained with 2\% uranyl acetate in 70\% methanol

399 followed by Reynold's lead citrate as described previously55. Tilt series were collected from $60^{\circ}$ to -

$40060^{\circ}$ (I.5 intervals) with a $200-\mathrm{kV}$ Tecnai F20 intermediate voltage electron microscopy

401 (https://www.fei.com/). Tomograms were reconstructed as described ${ }^{56}$. To generate models of

402 complicated thylakoid membranes, we used the autocontour command

403 (bio3d.colorado.edu/imod/doc/3dmodHelp/autox.html) of the $3 \mathrm{dmod}$ software package as explained

404 in Keith and Kang (2017) $)^{57}$.

\section{Acknowledgements}

406 We appreciate Dr. Xiaohong Zhuang (Chinese University of Hong Kong) for the atg5 407 and atg7 mutant lines, David Logan and David Macharel for kindly sharing Mito-GFP and 408 Friendly related seeds. We also thank Samantha Krasnodebski for help with generation of 409 Arabidopsis lines. This work was supported by grants from the Research Grants Council of 410 Hong Kong (GRFI4I26II6, GRFI4I2I0I9, C40I2-I6E, C4002-I7G, and AoE/ M-05/I2) and 411 Cooperative Research Program for Agriculture Science \& Technology Development (Project 412 No. 0109532019) Rural Development Administration, Republic of Korea to B.-H.K., and 413 Austrian Academy of Sciences and Austrian Science Fund (FWF): P32355 to Y.D.

\section{Author contributions}

415 J.M., Y.D., and B.-H.K. conceived and designed the experiments. J.M. performed the confocal 416 microscopy and stereomicroscopy. J.M., Z.L., and P.W. carried out electron 417 microscopy/tomography analysis. J.M. and J.F. prepared 3D tomographic models. J.M. and 418 W.M. performed immunoblot and pull-down experiments. J.Z., Y.Z., and N.G. did other 419 experiments. J.M., J.Z., Z.L., P.W., L.J., Y.D., and B.-H.K. analysed the data. J.M., Y.D., and B.420 H.K. wrote the paper.

\section{Competing interests}

422 The authors declare no competing interests. 


\section{References}

424

425

426

427

428

429

430

431

432

433

434

435

436

437

438

439

440

441

442

443

444

445

446

447

448

449

450

451

452

453

454

455

456

457

458

459

460

461

462

463

464

465

466

467

468

469

470

471

I. Youle, R. J. Mitochondria-Striking a balance between host and endosymbiont. Science (80-. ). 365, (2019).

2. Broda, M., Millar, A. H. \& Van Aken, O. Mitophagy: A Mechanism for Plant Growth and Survival. Trends Plant Sci. 23, 434-450 (20I8).

3. Pickles, S., Vigié, P. \& Youle, R. J. Mitophagy and Quality Control Mechanisms in Mitochondrial Maintenance. Curr. Biol. 28, RI70-RI85 (20I8).

4. Palikaras, K., Lionaki, E. \& Tavernarakis, N. Mechanisms of mitophagy in cellular homeostasis, physiology and pathology. Nat. Cell Biol. 20, I0I3-1022 (2018).

5. Dikic, I. Proteasomal and Autophagic Degradation Systems.

6. Anding, A. L. \& Baehrecke, E. H. Cleaning House: Selective Autophagy of Organelles. Dev. Cell 4 I, I0-22 (20I7).

7. Nguyen, T. N., Padman, B. S. \& Lazarou, M. Deciphering the Molecular Signals of PINK I / Parkin Mitophagy. xx, I-I2 (2016).

8. Montava-Garriga, L. \& Ganley, I. G. Outstanding Questions in Mitophagy: What We Do and Do Not Know. J. Mol. Biol. (2019) doi:I0.1016/j.jmb.2019.06.032.

9. Georgakopoulos, N. D., Wells, G. \& Campanella, M. The pharmacological regulation of cellular mitophagy. Nat. Chem. Biol. I3, I36-I46 (2017).

10. Wauer, T., Simicek, M., Schubert, A. \& Komander, D. Mechanism of phosphoubiquitin-induced PARKIN activation. Nature 524, 370-4 (20I5).

II. Lazarou, M. et al. The ubiquitin kinase PINKI recruits autophagy receptors to induce mitophagy. (2015) doi:10.1038/nature I4893.

12. Stephani, M. \& Dagdas, Y. Plant Selective Autophagy - Still an uncharted territory with a lot of hidden gems. J. Mol. Biol. (2019) doi:I0.1016/j.jmb.2019.06.028.

13. Li, F., Chung, T. \& Vierstra, R. D. AUTOPHAGY-RELATED I I plays a critical role in general autophagy- and senescence-induced mitophagy in Arabidopsis. Plant Cell 26, 788-807 (20I4).

14. Ashrafi, G. \& Schwarz, T. L. The pathways of mitophagy for quality control and clearance of mitochondria. Cell Death Differ. 20, 3I-42 (20I3).

I5. Zhou, Q. et al. Mitochondrial endonuclease G mediates breakdown of paternal mitochondria upon fertilization. Science (80-. ). 353, 394-399 (2016).

16. Klionsky, D. J. et al. Guidelines for the use and interpretation of assays for monitoring autophagy (3rd edition). Autophagy I 2, I-222 (2016).

17. Marshall, R. S. \& Vierstra, R. D. Autophagy: The Master of Bulk and Selective Recycling. Annu. Rev. Plant Biol. 69, I73-208 (2018).

18. Thompson, A. R., Doelling, J. H., Suttangkakul, A. \& Vierstra, R. D. Autophagic Nutrient Recycling in Arabidopsis Directed by the ATG8 and ATG 2 Conjugation Pathways. Plant Physiol. I 38, 2097-2 I I0 (2005).

19. Hanamata, S. et al. In vivo imaging and quantitative monitoring of autophagic flux in tobacco BY-2 cells. I-II (20I3).

20. Kellner, R., De la Concepcion, J. C., Maqbool, A., Kamoun, S. \& Dagdas, Y. F. ATG8 Expansion: A Driver of Selective Autophagy Diversification? Trends Plant Sci. (2016) doi:10.1016/j.tplants.2016.11.015.

21. Svenning, S., Lamark, T., Krause, K. \& Johansen, T. Plant NBRI is a selective autophagy substrate and a functional hybrid of the mammalian autophagic adapters NBRI and p62 / SQSTMI. 3, 993-1010 (201 I).

22. Kholmukhamedov, A., Schwartz, J. M. \& Lemasters, J. J. Isolated mitochondria infusion mitigates ischemia-reperfusion injury of the liver in rats: mitotracker probes and 
mitochondrial membrane potential. Shock 39, 543 (2013).

23. Yoshii, S. R., Kishi, C., Ishihara, N. \& Mizushima, N. Parkin mediates proteasomedependent protein degradation and rupture of the outer mitochondrial membrane. J. Biol. Chem. 286, I9630-19640 (201 I).

24. Lieber, T., Jeedigunta, S. P., Palozzi, J. M., Lehmann, R. \& Hurd, T. R. Mitochondrial fragmentation drives selective removal of deleterious $m t D N A$ in the germline. Nature 570, 380-384 (2019).

25. Twig, G. \& Shirihai, O. S. The interplay between mitochondrial dynamics and mitophagy. Antioxidants Redox Signal. I4, I939-195I (201 I).

26. El Zawily, A. M. et al. FRIENDLY Regulates Mitochondrial Distribution, Fusion, and Quality Control in Arabidopsis. Plant Physiol. 166, 808-828 (20I4).

27. Ka, K., Mai, K., Gao, P. \& Kang, B. Electron Microscopy Views of Dimorphic Chloroplasts in C4 Plants. I I, I-7 (2020).

28. Mizushima, N. A brief history of autophagy from cell biology to physiology and disease. Nat. Cell Biol. 20, 52I-527 (20I8).

29. Pohl, C. \& Dikic, I. Cellular quality control by the ubiquitin-proteasome system and autophagy. Science (80-. ). 366, 818-822 (2019).

30. Levine, B. \& Kroemer, G. Biological Functions of Autophagy Genes: A Disease Perspective. Cell I 76, II-42 (20I9).

31. Wade Harper, J., Ordureau, A. \& Heo, J. M. Building and decoding ubiquitin hains for mitophagy. Nat. Rev. Mol. Cell Biol. 19, 93-108 (2018).

32. Sato, M. \& Sato, K. Degradation of Paternal Mitochondria. Science (80-. ). 37, II4II I 44 (20I I).

33. Cummins, J. M. et al. Postfertilization Autophagy of Sperm. I, I I44-I I 48 (20 I I).

34. Malka, F. et al. Separate fusion of outer and inner mitochondrial membranes. EMBO Rep. 6, 853-859 (2005).

35. Arimura, S. Fission and fusion of plant mitochondria, and genome maintenance. Plant Physiol. pp.01025.20I7 (2017) doi:I0.I I04/pp. I7.01025.

36. Sheahan, M. B., McCurdy, D. W. \& Rose, R. J. Mitochondria as a connected population: Ensuring continuity of the mitochondrial genome during plant cell dedifferentiation through massive mitochondrial fusion. Plant J. 44, 744-755 (2005).

37. Seguí-Simarro, J. M. \& Staehelin, L. A. Cell cycle-dependent changes in Golgi stacks, vacuoles, clathrin-coated vesicles and multivesicular bodies in meristematic cells of Arabidopsis thaliana: A quantitative and spatial analysis. Planta 223, 223-236 (2006).

38. Wang, Y. et al. Kinetics and specificity of paternal mitochondrial elimination in Caenorhabditis elegans. Nat. Commun. 7, I-I5 (2016).

39. Wei, Y., Chiang, W. C., Sumpter, R., Mishra, P. \& Levine, B. Prohibitin 2 Is an Inner Mitochondrial Membrane Mitophagy Receptor. Cell I 68, 224-238.el0 (20I7).

40. Cox, R. T. \& Spradling, A. C. Clueless, a conserved Drosophila gene required for mitochondrial subcellular localization, interacts genetically with parkin. DMM Dis. Model. Mech. 2, 490-499 (2009).

4I. Wang, Z. H., Clark, C. \& Geisbrecht, E. R. Drosophila clueless is involved in Parkindependent mitophagy by promoting VCP-mediated Marf degradation. Hum. Mol. Genet. 25, 1946-1964 (2016).

42. Pla-Martín, D. et al. CLUH granules coordinate translation of mitochondrial proteins with mTORCI signaling and mitophagy. EMBO J. 39, I-23 (2020).

43. Sheard, K. M., Thibault-Sennett, S. A., Sen, A., Shewmaker, F. \& Cox, R. T. Clueless forms dynamic, insulin-responsive bliss particles sensitive to stress. Dev. Biol. 459, I49-160 (2020).

44. Spinelli, J. B. \& Haigis, M. C. The multifaceted contributions of mitochondria to 
cellular metabolism. Nat. Cell Biol. 20, 745-754 (20I8).

45. Raghavendra, A. S., Padmasree, K. \& Saradadevi, K. Interdependence of photosynthesis and respiration in plant cells: interactions between chloroplasts and mitochondria. Plant Sci. 97, I-I4 (1994).

46. Van Lis, R. \& Atteia, A. Control of mitochondrial function via photosynthetic redox signals. Photosynth. Res. 79, I33-I 48 (2004).

47. Garmash, E. V. et al. Expression profiles of genes for mitochondrial respiratory energy-dissipating systems and antioxidant enzymes in wheat leaves during deetiolation. J. Plant Physiol. 21 5, II0-12I (2017).

48. Zhuang, X. et al. A BAR-Domain Protein SH3P2, Which Binds to Phosphatidylinositol 3-Phosphate and ATG8, Regulates Autophagosome Formation in Arabidopsis. I-2I (20I3) doi:I0.I I05/tpc. II3.II8307.

49. Paszkiewicz, G., Gualberto, J. M., Benamar, A., Macherel, D. \& Logan, D. C. Arabidopsis seed mitochondria are bioenergetically active immediately upon imbibition and specialize via biogenesis in preparation for autotrophic growth. Plant Cell 29, 109-128 (2017).

50. Zhuang, X. et al. ATG9 regulates autophagosome progression from the endoplasmic reticulum in \&lt;em\&gt;Arabidopsis\&lt;/em\&gt; Proc. Natl. Acad. Sci. I I4, E426 LP-E435 (2017).

5I. Marshall, R. S., Li, F., Gemperline, D. C., Book, A. J. \& Vierstra, R. D. Autophagic Degradation of the 26S Proteasome Is Mediated by the Dual ATG8/Ubiquitin Receptor RPNIO in Arabidopsis. Mol. Cell 58, I053-1066 (20I5).

52. Chung, T., Phillips, A. R. \& Vierstra, R. D. ATG8 lipidation and ATG8-mediated autophagy in Arabidopsis require ATG 2 expressed from the differentially controlled ATGI2A and ATGI2B loci. Plant J. 62, 483-493 (2010).

53. Kang, B. H. Electron microscopy and high-pressure freezing of arabidopsis. Methods in Cell Biology vol. 96 (Elsevier Inc., 2010).

54. Wang, P., Chen, X., Goldbeck, C., Chung, E. \& Kang, B. H. A distinct class of vesicles derived from the trans-Golgi mediates secretion of xylogalacturonan in the root border cell. Plant J. 92, 596-610 (2017).

55. Liang, Z. et al. Thylakoid-bound polysomes and a dynamin-related protein, FZL, mediate critical stages of the linear chloroplast biogenesis program in greening arabidopsis cotyledons. Plant Cell 30, I476-I495 (2018).

56. Toyooka, K. \& Kang, B.-H. Reconstructing Plant Cells in 3D by Serial Section Electron Tomography BT - Plant Cell Morphogenesis: Methods and Protocols. in (eds. Žárský, V. \& Cvrčková, F.) I59-I 70 (Humana Press, 20 I4). doi:I0.I007/978-I-62703-643$6 \_13$.

57. Mai, K.K.K. \& Kang, B.-H. Semiautomatic Segmentation of Plant Golgi Stacks in Electron Tomograms Using 3dmod. - Plant Protein Secretion: Methods and Protocols. in (eds. Liwen Jiang) 97-104 (Springer Science+Business Media, 2017). doi: 10.1007/978-I-4939-7262-3_8 


\section{Main Figures}

a

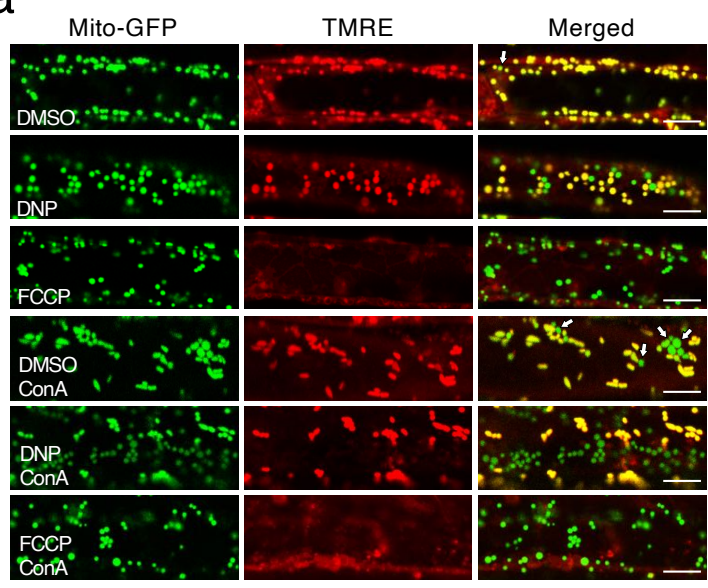

C

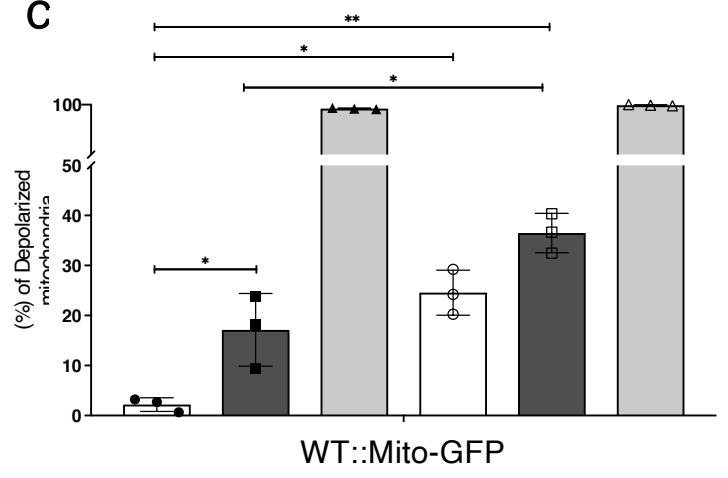

b

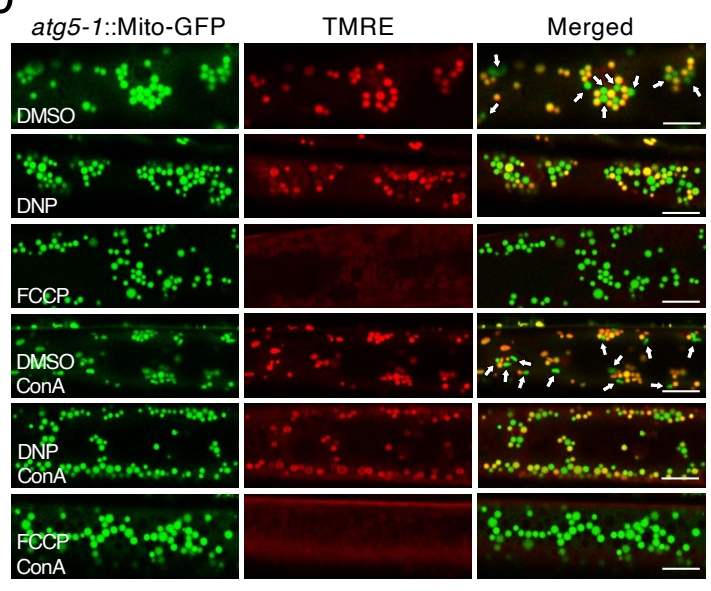

d

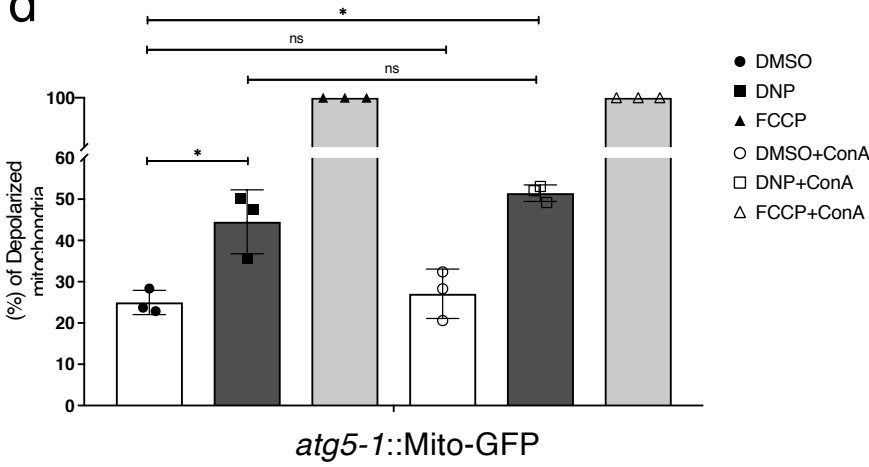

Figure I. Arabidopsis root cells accumulate depolarized mitochondria upon uncoupler treatments.

a,b, Uncoupler treatment induced mitochondria depolarization. Confocal micrographs of wild-type (a) and atg5-I (b) root cells expressing a mitochondrion-targeted GFP (Mito-GFP) after uncoupler treatment. Mitochondria were prestained with TMRE. Normal mitochondria exhibit yellow fluorescence while depolarized mitochondria exhibit green fluorescence (arrows) in DMSO or DMSO + ConA panels in the merged image columns. Note that most mitochondria are round. Scale bars, $8 \mu \mathrm{m}$. c,d, Histograms illustrating the percentage of depolarized mitochondria in wild type (WT) and atg5-I root cells expressing Mito-GFP at each treatment conditions. Bars represent the mean $( \pm S D)$ of three biological replicates, each generated with three technical replicates. About 500 mitochondria from 10 cells (five root samples) were counted per condition. Asterisks $(*)$ denote significant differences in depolarized mitochondria percentages relative to DMSO control group under each condition (unpaired t-test, ${ }^{*}<0.05,{ }^{*}{ }^{*}<0.01$, ns, no significant difference). 
a
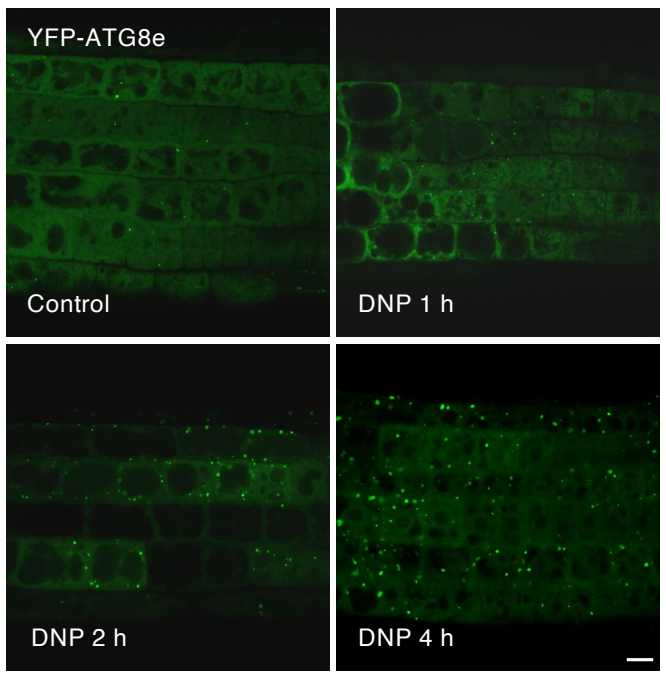

C
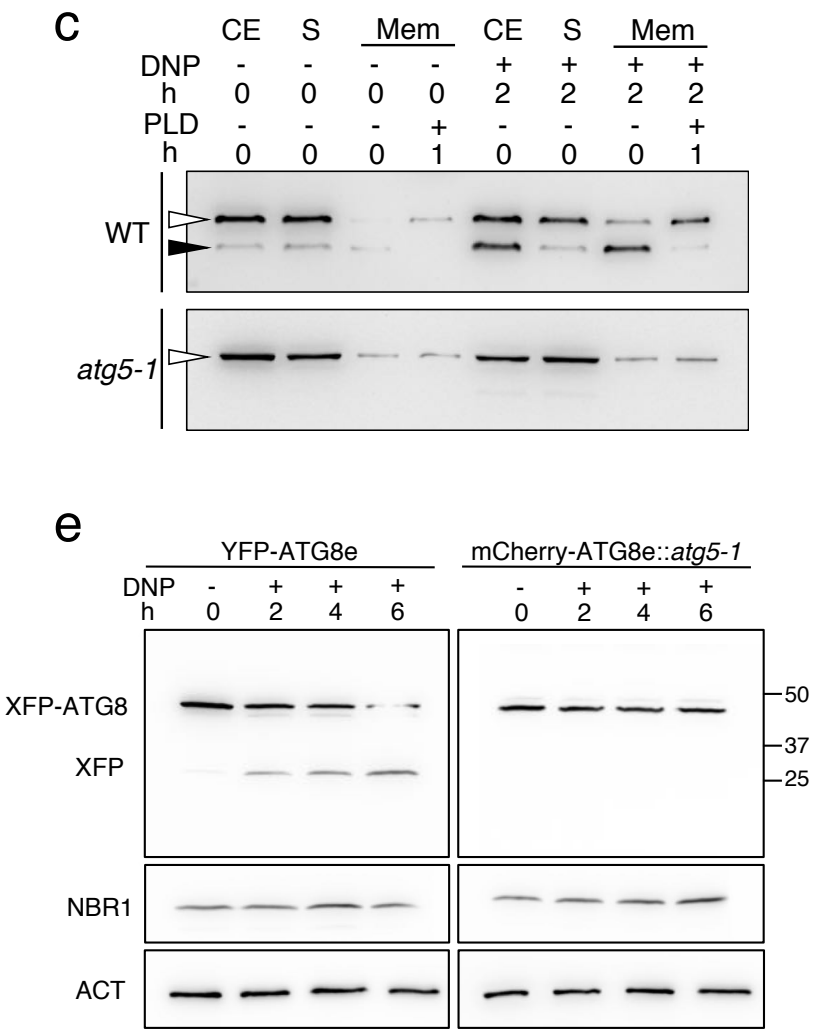

b

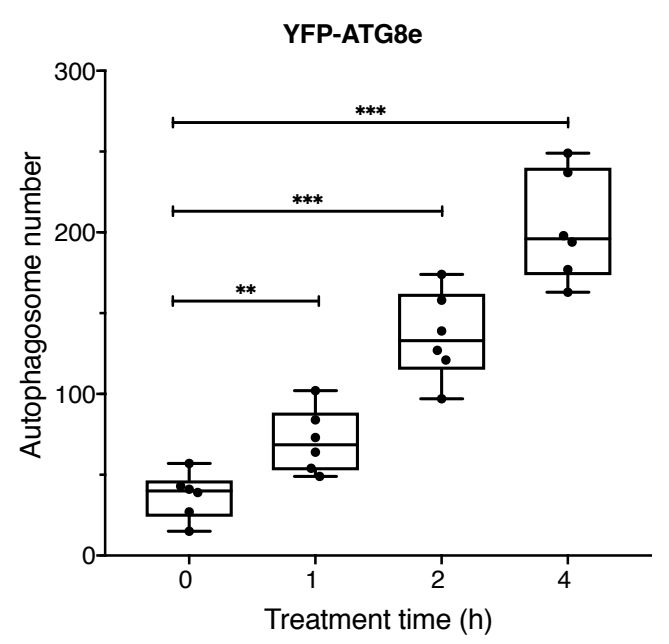

d

WT

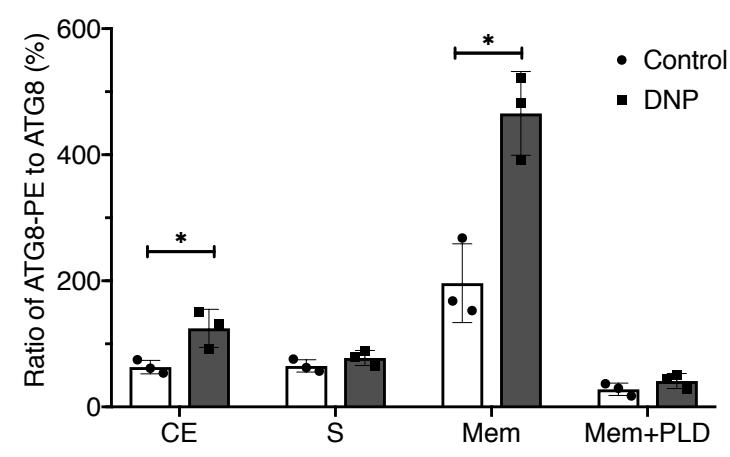

f

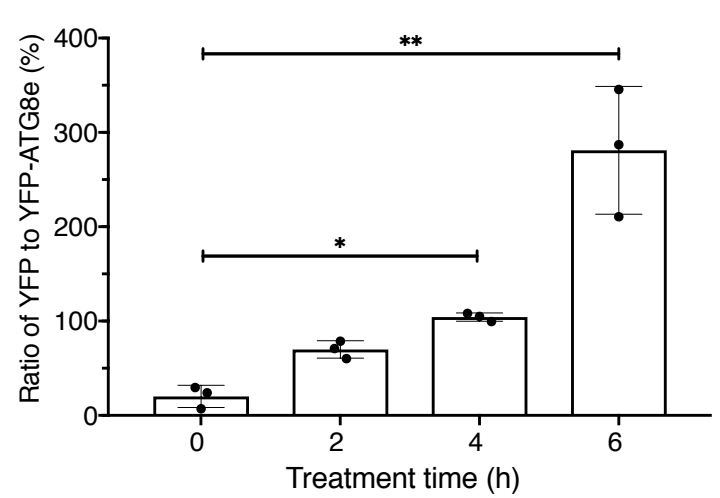


Figure 2. Uncoupler treatments induce autophagy.

a, DNP treatment induces autophagosome formation. Arabidopsis YFP-ATG8e seedlings were incubated in DNP solution for varying periods $(0-4 \mathrm{~h})$ before imaging. $\mathbf{b}$, Quantification of the number of autophagosomes from more than five independent root samples (unpaired t-test, $* * \mathrm{p}<0.0 \mathrm{I}$, *** $p<0.00 \mathrm{I}$ ). Scale bars, $8 \mu \mathrm{m}$. c, Uncoupler treatment activates ATG8 lipidation. Protein crude extracts (CE) were prepared from Arabidopsis root cells following incubation in DNP for 2 hours. Soluble (S) and membrane (Mem) fractions were separated and examined by immunoblot analysis with an anti-ATG8 antibody. White arrowheads mark ATG8. An additional polypeptide recognized by the antibody is enriched in the membrane fraction when cells are incubated with DNP (black arrowhead). d, Histograms illustrating polypeptide intensity ratios of lipidated ATG8 to free ATG8 in (c). Bars represent the mean $( \pm S D)$ of three biological replicates. e, ATG8 cleavage assays of DNP treated WT and atg5-I seedlings expressing YFP-ATG8e or mCherry-ATG8e, respectively. Protein extracts were prepared from Arabidopsis seedlings exposed to DNP $(50 \mu \mathrm{M})$ for the indicated time periods and subjected to immunoblot analysis with anti-GFP or anti-mCherry antibodies. NBRI and Actin were used as control. f, Histograms illustrating the polypeptide intensity ratios of free YFP to YFP-ATG8e in (d). Bars represent the mean $( \pm S D)$ of three biological replicates. (unpaired t-test, ${ }^{*} p<0.05,{ }^{* *} p<0.01$ ). 

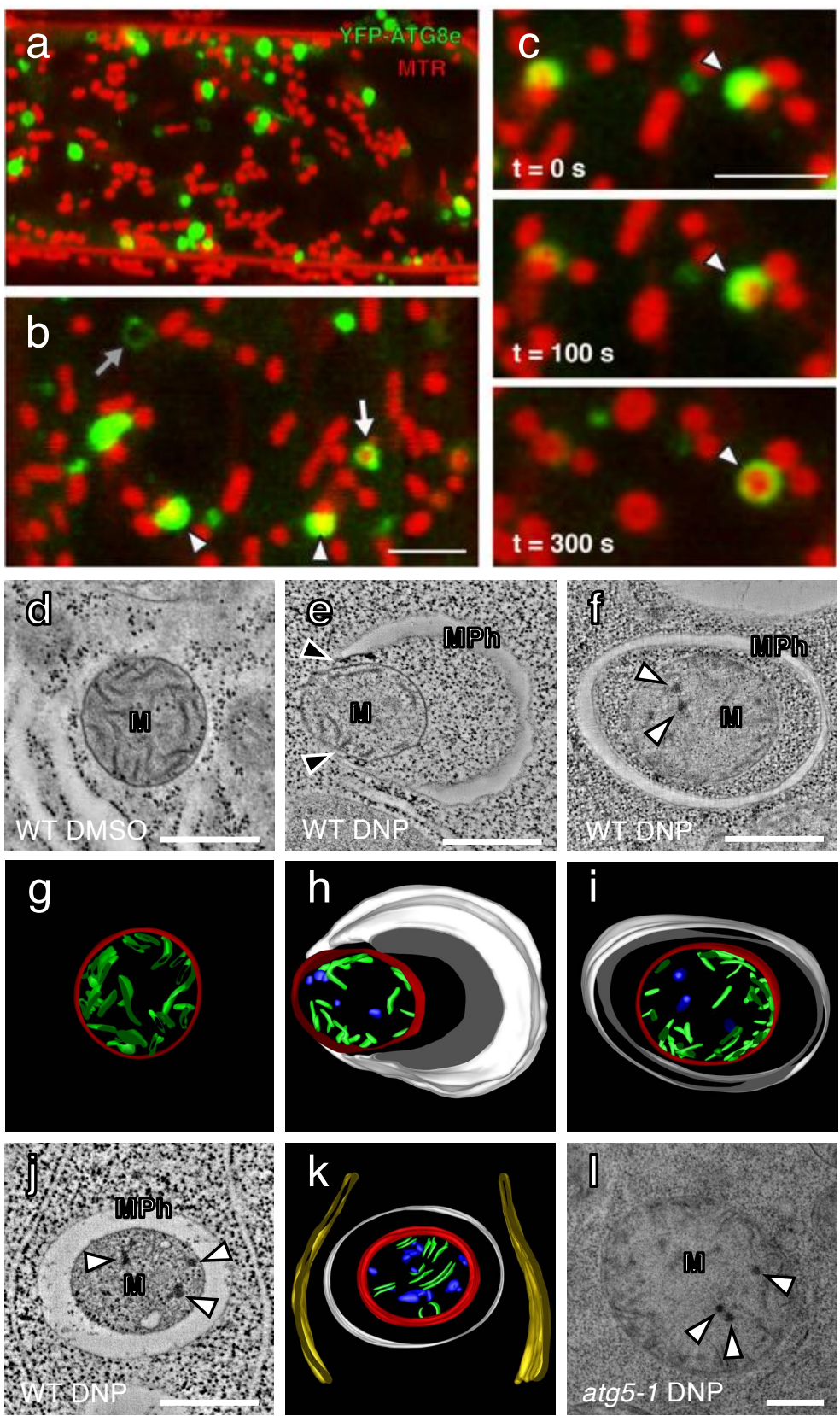
Figure 3. Depolarized mitochondria are selectively engulfed by autophagosomes in uncouplertreated Arabidopsis root cells.

a,b, DNP treatment induces mitophagy in Arabidopsis root cells. Confocal micrographs of Arabidopsis root cells expressing YFP-ATG8e stained with MitoTracker Red (MTR) and incubated with DNP for I hour. The mitochondria that associate with YFP-ATG8e are indicated with the arrowheads in panel (b). The mitochondria that are completely engulfed by ATG8e fluorescence is marked with a white arrow. Empty YFP fluorescence circles were also observed (grey arrow in panel b). Scale bars, $5 \mu \mathrm{m}$. c, Time lapse imaging of a mitophagy event in an Arabidopsis root tip cell treated with DNP. The mitochondrion is engulfed by YFPATG8e over 5 minutes. Scale bar, $5 \mu \mathrm{m}$. d-f,j,l, Transmission electron micrographs of mitochondria (M) in Arabidopsis WT or atg5-I root cells incubated with DMSO or DNP. Mitochondria phagophores (MPh) assemble in the vicinity of the mitochondria. Note that the phagophore tips (black arrow) are in contact with the mitochondrial surface in (e). g-i,k, Three-dimensional models of the mitophagosome (MPh) and its mitochondrial cargo (M) based on the tomogram in (d-f,j,l). Mitophagosome (white), mitochondria outer membrane (red), mitochondria cristae (green), damaged cristae formed aggregates (blue) and ER (yellow) are modeled. White arrows indicate dark aggregates in the matrix of compromised mitochondria. Scale bars, 500 $\mathrm{nm}$. 
a

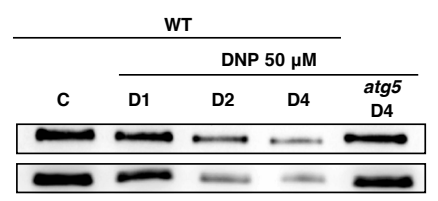

는 - - - -

- - - - -
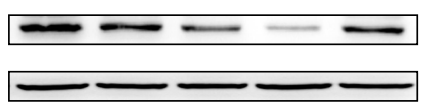

b
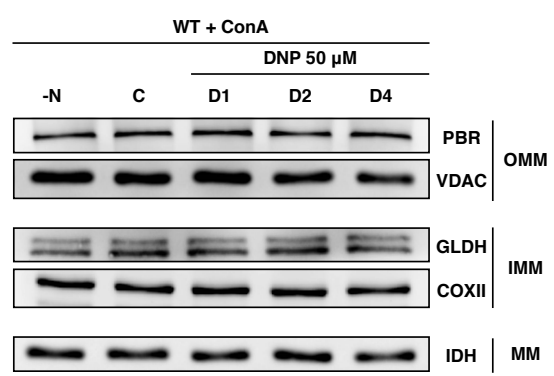

d

$=2020$ SMT1 ER
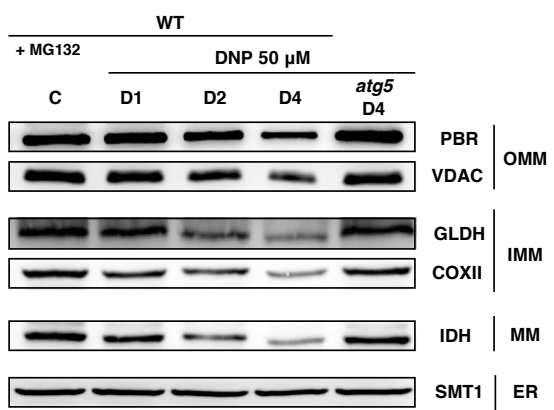

C
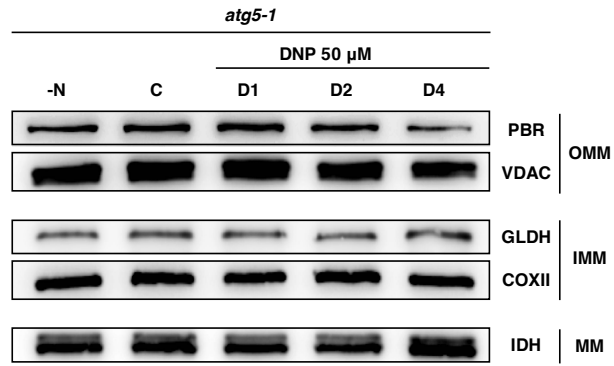

$=$ SMT1 I ER
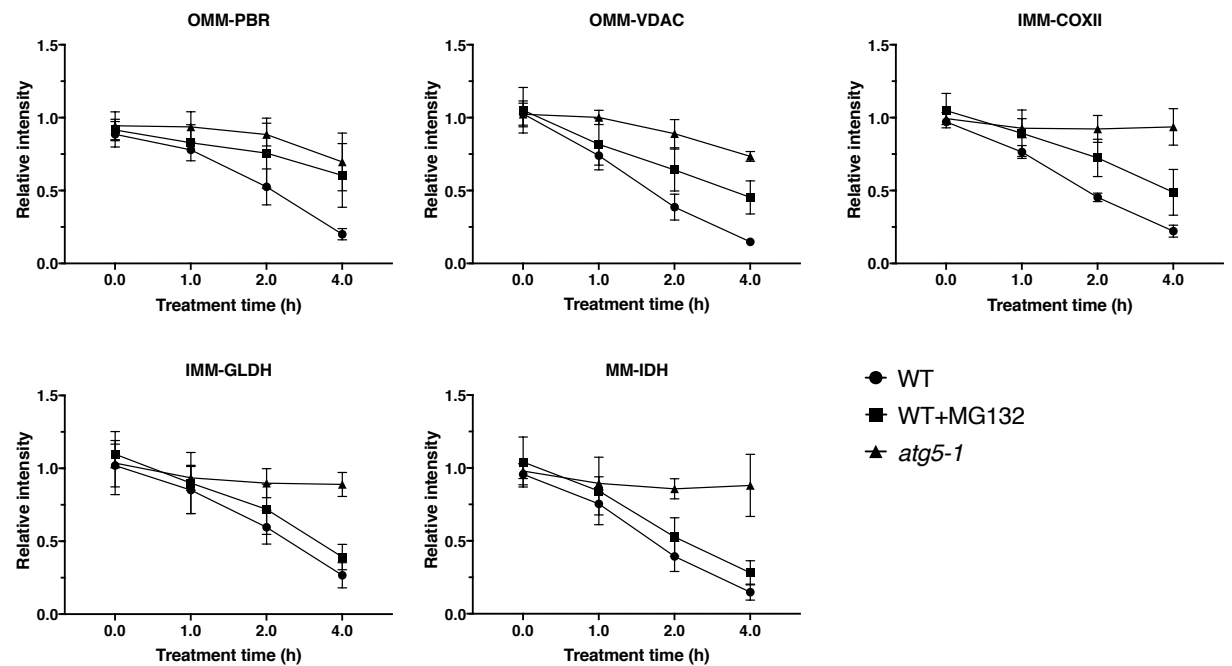

e
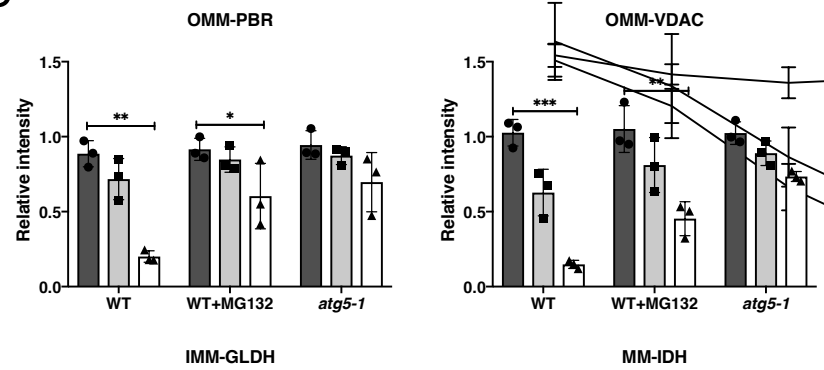

- WT

- WT+MG132

× $\operatorname{atg5-1}$

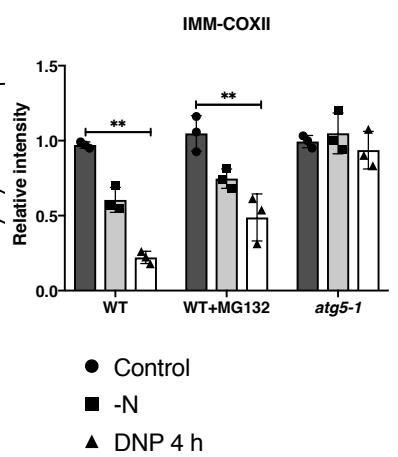

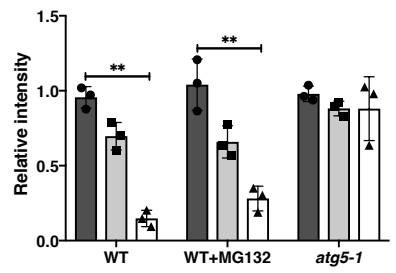




\section{Figure 4. ATG5-dependent degradation of mitochondrial proteins in uncoupler-treated}

Arabidopsis root cells.

a-c, Immunoblot blot analyses of uncoupler treatment induced mitochondrial protein degradation in Arabidopsis WT and atg5-I seedlings. WT and atg5-I mutant Arabidopsis roots were incubated in DNP solution for I to 4 hours (DI-D4) or nitrogen starvation (-N) solutions for I day. Mitochondrial outer membrane, mitochondrial matrix, and endomembrane fractions were isolated and subjected to immunoblot analyses. For outer mitochondrial membrane (OMM) proteins, PBR and VDACI, for inner mitochondrial membrane (IMM) COXII and GLDH, and for mitochondrial matrix IDH were analysed. Concanamycin A (ConA) was added to the treatment solution to test for vacuolar function in mitochondrial protein degradation. Proteasome inhibitor MGI32 was added to test the involvement of proteasomes in the recycling of mitochondrial membrane proteins (c). Note that an ER protein, SMTI was not affected by DNP treatment. Equal amounts of protein extracts were analysed in the immunoblots shown. d, Line charts illustrating degradation rates of OMM proteins (PBR and VDACI), IMM proteins (COXII and GLDH), and MM protein (IDH) in WT treated with DNP (WT), atg5-I treated with DNP (atg5-I), and WT treated with DNP and MGI32 (WT+MGI32). e, Histograms illustrating the levels of mitochondria membrane proteins under the three treatment conditions, DMSO $4 \mathrm{~h}$ (Control), DNP $4 \mathrm{~h}$, and nitrogen starvation (-N) in WT and atg5-I root cells. The polypeptide intensity values were normalized to that of the loading control (SMTI). Bars represent the mean ( \pm SD) and the asterisks $\left({ }^{*}\right)$ indicate decreases in polypeptide readouts significantly from that of the control (C) point (unpaired ttest, $* \mathrm{P}<0.05$, $* * \mathrm{P}<0.0 \mathrm{I}$, ***P $<0.01$ ). 

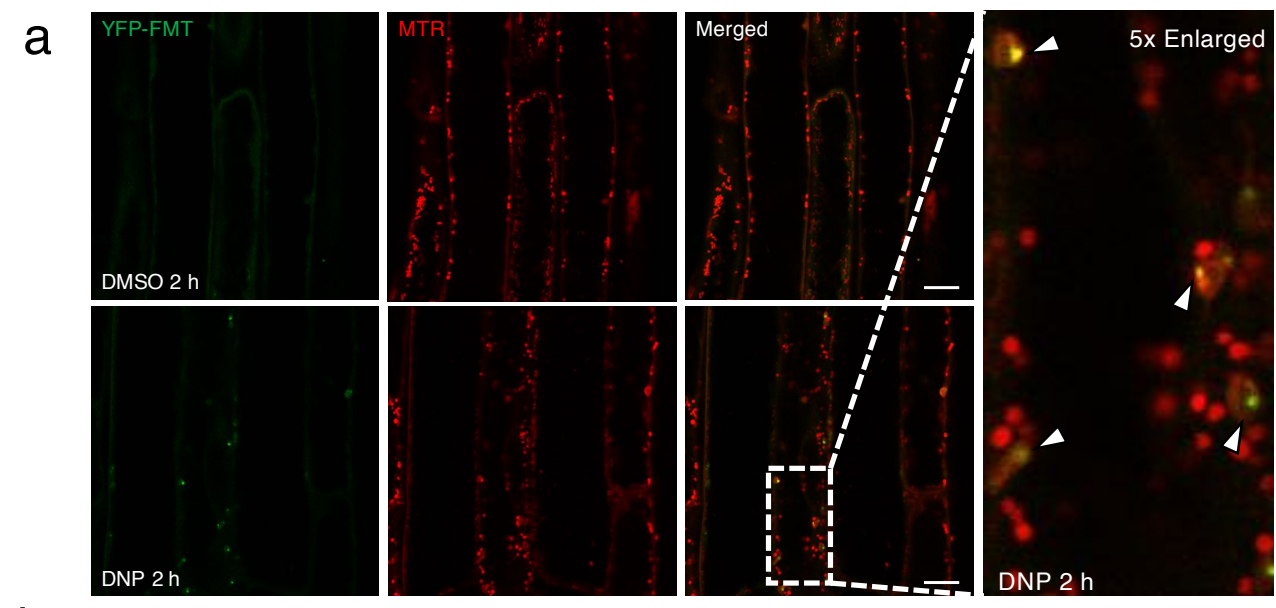

b
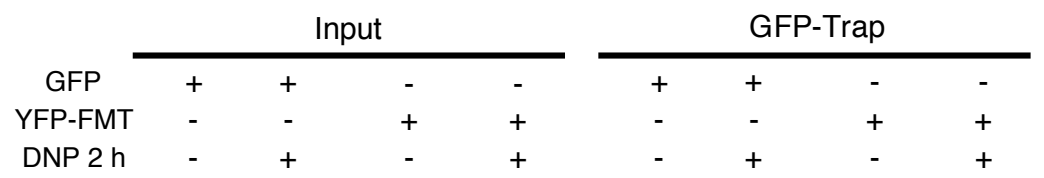

IB: GFP
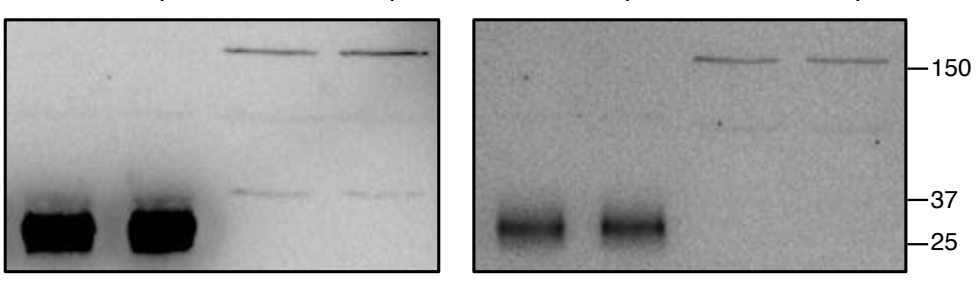

IB: ATG8
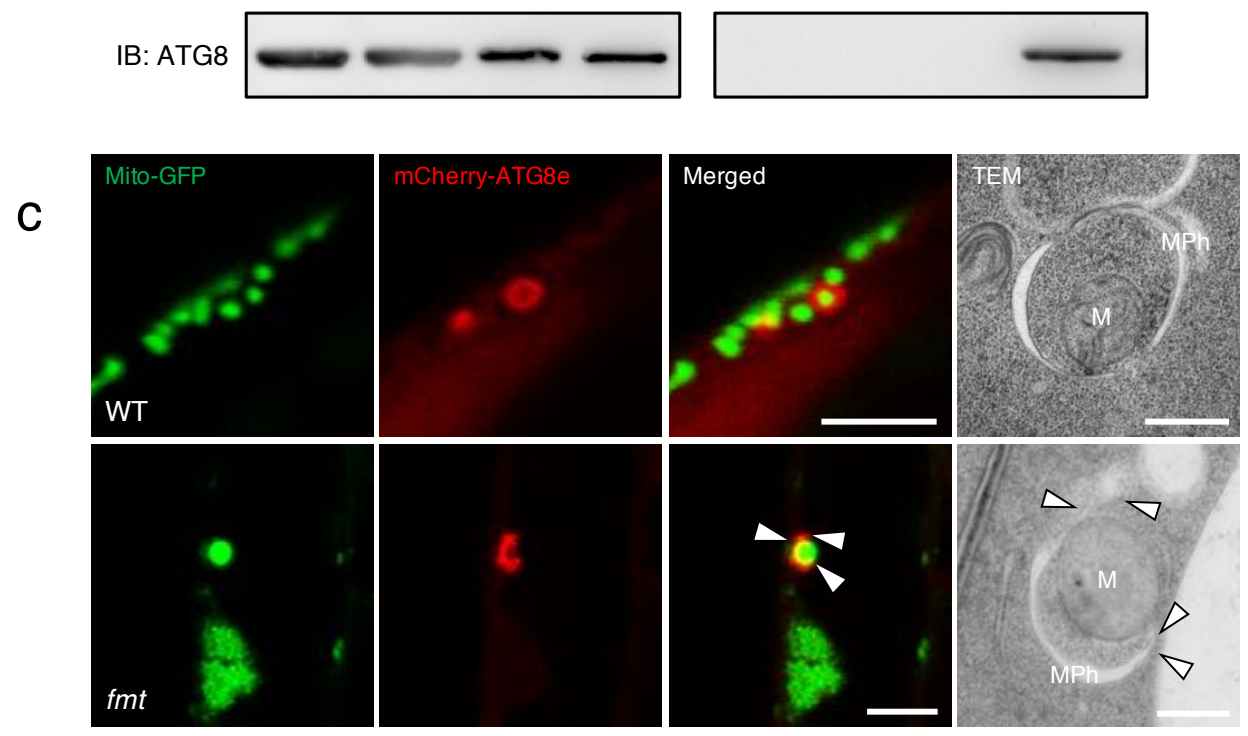
Figure 5. Friendly associates with damaged mitochondria and ATG8 upon uncoupler treatment.

a, DNP treatment induces recruitment of Friendly (FMT) to damaged mitochondria. Confocal micrographs of Arabidopsis root cells expressing a YFP-tagged FMT (YFP-FMT) prestained with MTR. FMT-YFP seedlings were incubated with DMSO or DNP for I hour prior to imaging. Scale bars, $8 \mu \mathrm{m}$. $\mathbf{b}$, FMT associates with ATG8 upon DNP treatment. Arabidopsis root cells expressing YFP-FMT were incubated with DMSO or DNP for 2 hours and then subjected to immunoprecipitation with GFP-trap followed by immunoblotting with indicated antibodies. c, Confocal micrographs of Arabidopsis WT and fmt root cells expressing mitochondria-targeted GFP (Mito-GFP) and mCherry-targeted ATG8e (mCherry-ATG8e). WT or fmt plants seedlings were incubated with DNP for I hour prior to imaging. Scale bars, $8 \mu \mathrm{m}$. Transmission electron microscopy (TEM) photos show mitochondrial phagophores $(\mathrm{MPh})$ assemble in the vicinity of the mitochondria (M) under the condition for the experiment in (a). Arrowhead point to defective phagophores in confocal and TEM micrographs. Scale bars, $500 \mathrm{~nm}$. 
a
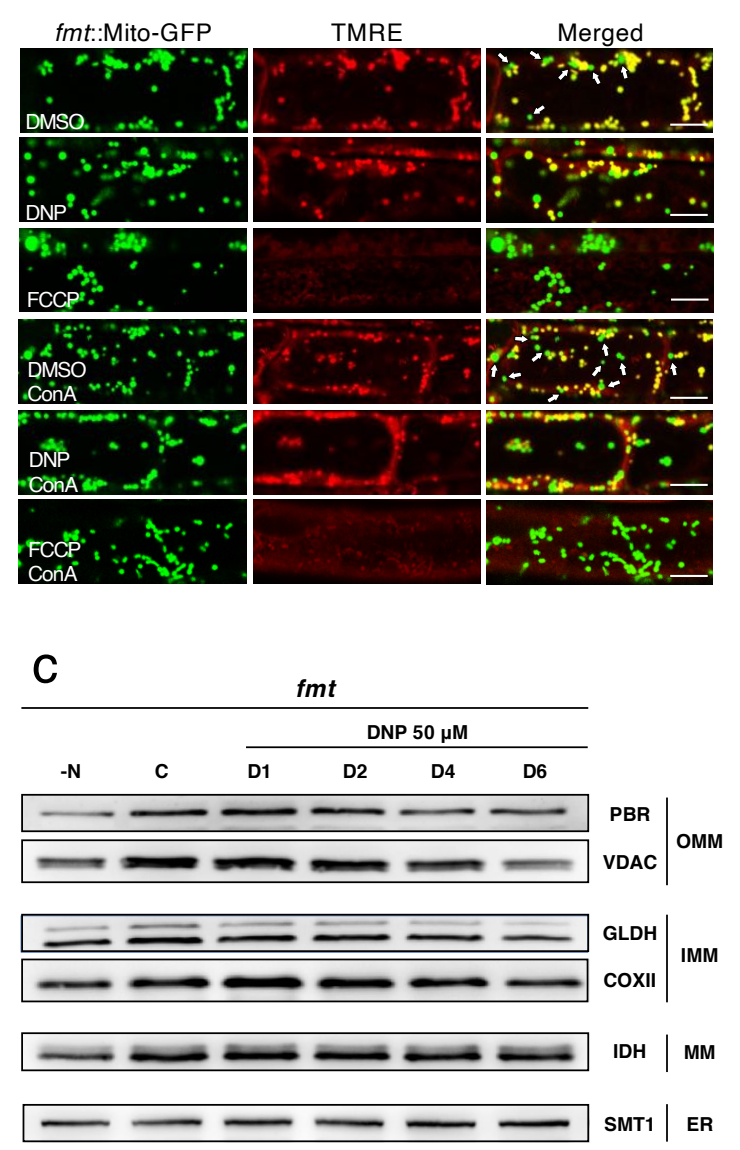

b
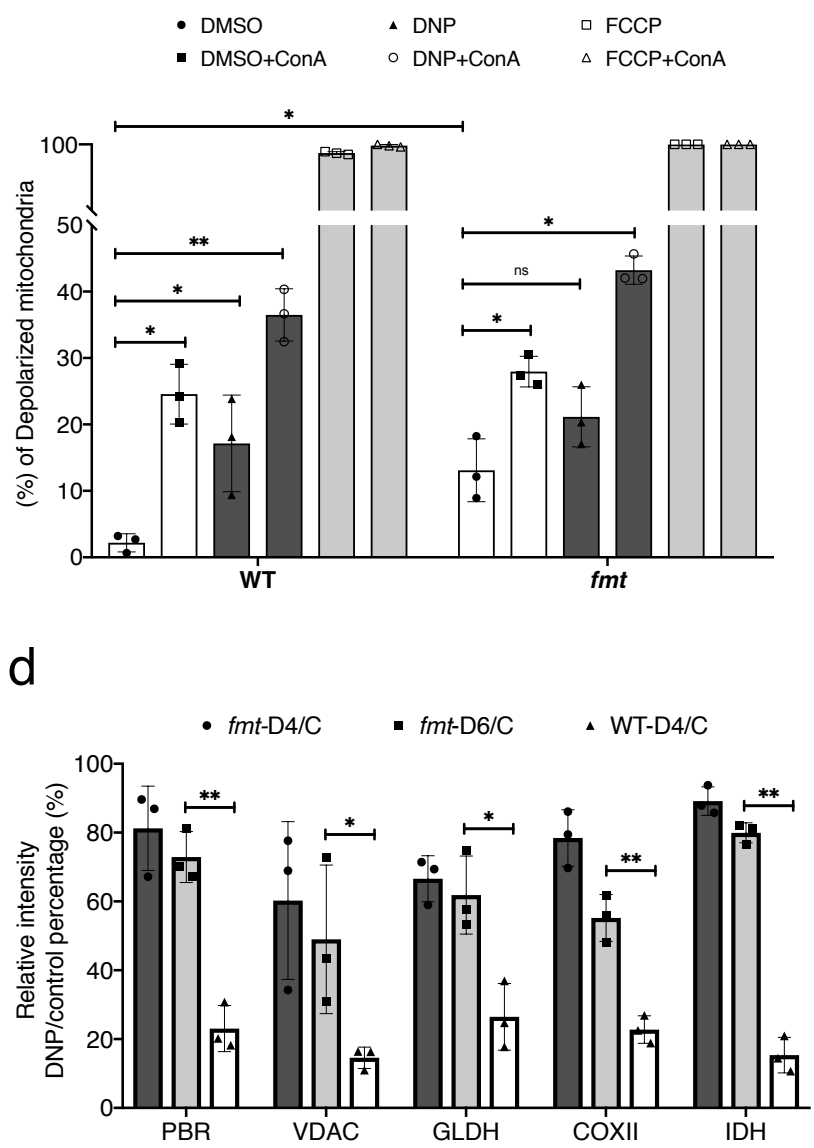

Figure 6. Arabidopsis friendly mutant have defects in clearance of depolarized mitochondria.

a, Uncoupler treatment induced mitochondria depolarization in Arabidopsis friendly mutant (fmt). Confocal micrographs of fmt root cells expressing a mitochondrion-targeted GFP (Mito-GFP), stained with TMRE. Normal mitochondria exhibit yellow fluorescence and depolarized mitochondria exhibit green florescence, denoted with arrows in DMSO or DMSO +ConA panels in the merged image column. Arabidopsis seedings were incubated with DMSO, DNP or FCCP with or without ConA for I hour prior to imaging. Scale bars, $8 \mu \mathrm{m}$. b, Histograms illustrating the percentage of depolarized mitochondria for each treatment conditions. Bars represent the mean $( \pm S D)$ of three biological replicates. About 500 mitochondria from 10 cells (five root samples) were counted per condition. An asterisk $(*)$ represents a significant difference of depolarized mitochondria percentage in each treatment relative to DMSO control group (unpaired t-test, $*_{p}<0.05$, $* * p<0.01$ ). c, Uncoupler treatment induced mitochondrial protein degradation in fmt mutant. Arabidopsis fmt mutant roots were incubated in DNP solutions for I hour to 6 hours (DI-D6) or nitrogen starvation (N-) solutions for I day. For outer mitochondrial membrane (OMM) proteins, PBR and VDACI, for inner mitochondrial membrane (IMM) COXII and GLDH, and for mitochondrial matrix IDH were examined with immunoblot analysis. Note that an ER protein, SMTI, was not affected by DNP treatment. d, Histograms illustrating the levels of mitochondrial membrane protein degradation for the DNP treatment (DNP $4 \mathrm{~h}$, DNP $6 \mathrm{~h}$ ) in WT and fmt root cells. The polypeptide intensity values were normalized with that of the loading control (SMTI) and the percentage of DNP treatment group to control group are quantitated. Bars represent the mean $( \pm S D)$ and the asterisks $(*)$ indicate the significantly difference in polypeptide readouts between $\mathrm{fmt}$ and WT groups (unpaired t-test, $* \mathrm{P}<0.05, * * \mathrm{P}<0.0 \mathrm{I}$ ). 
a
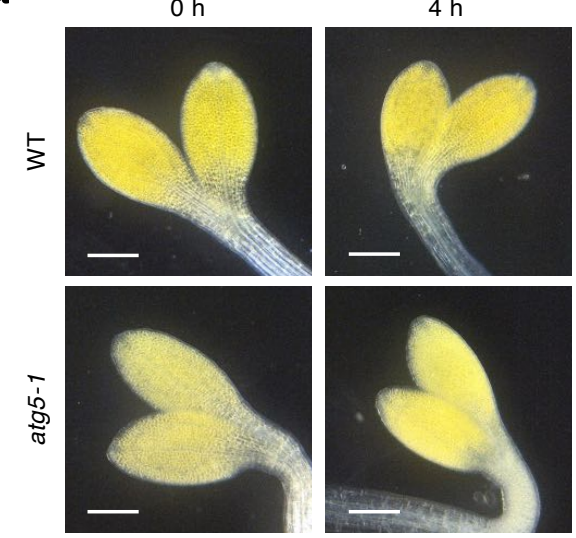

b

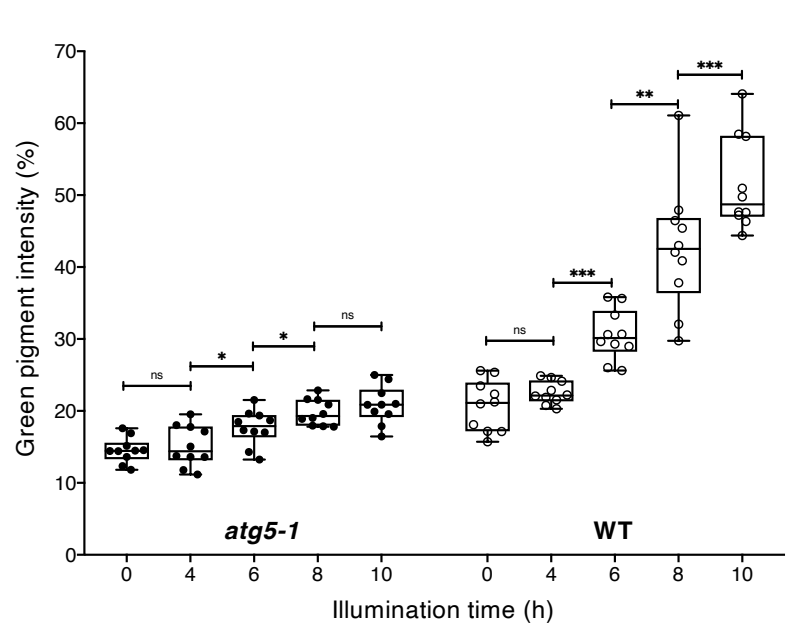

C

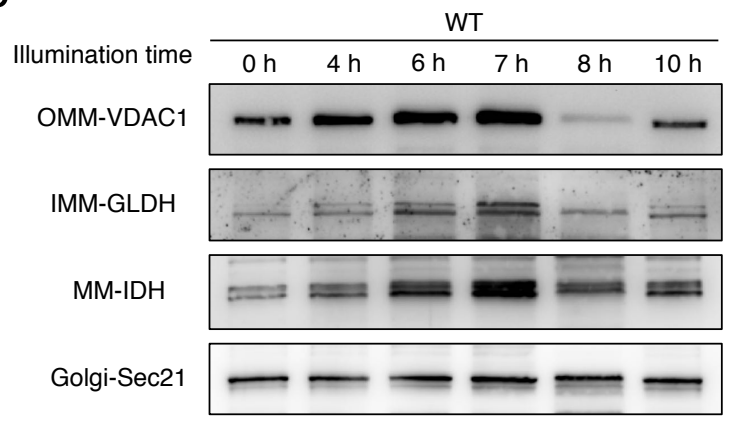

$8 \mathrm{~h}$
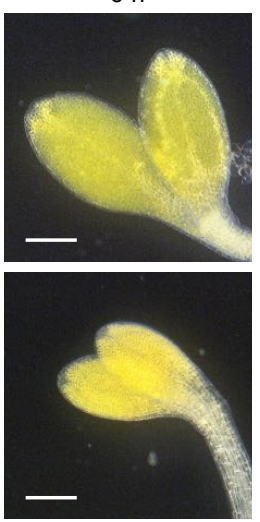
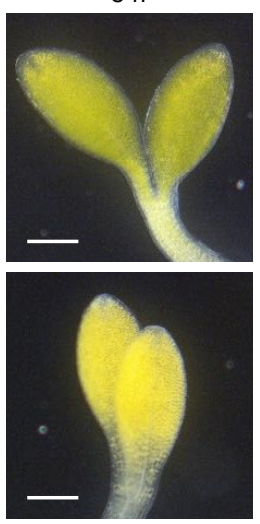

$12 \mathrm{~h}$
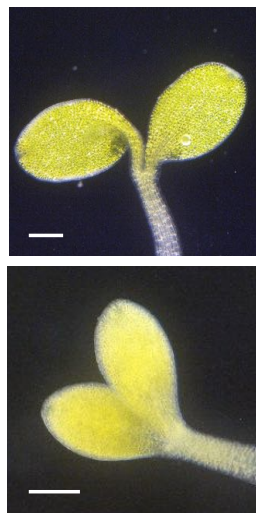

d

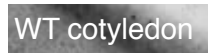

M

M

M

$\checkmark$

V.

6 h Illumination

e

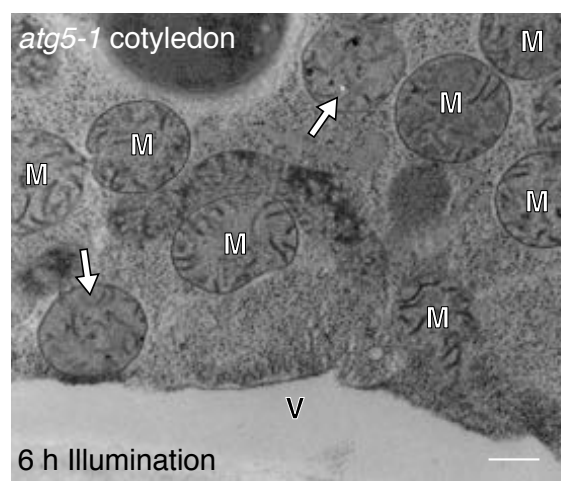


Figure 7. Mitophagy is triggered during de-etiolation of Arabidopsis seedlings.

a, Cotyledon greening after light exposure in WT and atg5-I Arabidopsis. Darkfield stereo microscopy photos showing Arabidopsis WT and atg5-I mutant cotyledon at multiple time points $(0-12 \mathrm{~h})$ after illumination. Arabidopsis seedlings were grown darkness before the experiment. Scale bars, I mm. b, Quantification of the green pigments in cotyledons after light exposure. The green colour of ten Arabidopsis cotyledons for each time points were calculated from their photos and normalized against the dark background. (unpaired t-test, ${ }^{*} \mathrm{p}<0.05$, ${ }^{* *} \mathrm{p}<0.0 \mathrm{I},{ }^{* * *} \mathrm{p}<0.00 \mathrm{I}$, ns, no significant difference). c, Immunoblot analyses of mitochondrial proteins in Arabidopsis seedlings during de-etiolation. For outer mitochondrial membrane (OMM) proteins, PBR and VDACI, for inner mitochondrial membrane (IMM) COXII and GLDH, and for mitochondrial matrix IDH were analysed as representative proteins. An Arabidopsis Golgi protein, coatomer subunit gamma (Sec2I), was employed as the loading control. d,e, TEM images of a cluster of mitochondria (M) near the vacuole (V) in Arabidopsis WT and atg5-I cotyledon cell after 6 hours of illumination. Mitochondria were seen to be surrounded by mitophagosome (MPh) in WT. By contrast, compromised mitochondria with dark aggregates were abundant in atg5-I (arrows in e) but no mitophagosomes were associated with them. Scale bars, $500 \mathrm{~nm}$. 